\title{
OTIMIZAÇÃo DA PREPARAÇÃo DE ELETRODO DE PASTA DE CARBONO CONTENDO RIBOFLAVINA IMOBILIZADA EM SUPORTE INORGÂNICO
}

\author{
Arnaldo César Pereira e Lauro Tatsuo Kubota*
}

Instituto de Química, Universidade Estadual de Campinas, CP 6154, 13084-971 Campinas - SP

Recebido em 19/9/03; aceito em 27/1/04; publicado na web em 17/6/04

\begin{abstract}
OPTIMIZATION OF THE CARBON PASTE ELECTRODE PREPARATION CONTAINING RIBOFLAVIN IMMOBILIZED ON AN INORGANIC SUPPORT. The aim of this work was to optimize the preparation of electrodes with riboflavin (RF) immobilized on a silica surface modified with niobium oxide and carbon paste. Electrode preparation was optimized employing a factorial design consisting of two levels and three factors. The electrochemical properties of immobilized RF were investigated by cyclic voltammetry. The factorial analysis was carried out analysing the current intensity $\left(\mathrm{I}_{\mathrm{pa}}\right)$. It was possible to optimize the electrode to get the best reversibility in the redox process, i. e. the lowest separation between anodic and cathodic peak potentials and a current ratio close to unity. The concentration of supporting electrolyte has a small effect. The proportion has the highest effect and the interaction factor between proportion and mixture has also a significant effect on the current intensity.
\end{abstract}

Keywords: carbon paste electrode; optimization of electrochemical properties; factorial design.

\section{INTRODUÇÃO}

A imobilização de mediadores de elétrons sobre superfícies para produzir eletrodos quimicamente modificados para uso em eletroanálise vem sendo amplamente investigada durante as últimas duas décadas ${ }^{1-4}$. Eletrodos modificados com quinonas, flavinas e outras classes de compostos quinóicos, tais como fenoxazina, estão sendo amplamente explorados para o desenvolvimento de sensores ${ }^{5-8}$.

A importância de flavinas em catálises enzimáticas tem motivado os estudos do comportamento eletroquímico de monocamadas ou agregados contendo flavinas sintéticas ${ }^{9}$. A flavina nucleotídeo tem a habilidade para transferir um ou dois elétrons e promover a redução de oxigênio molecular ${ }^{10}$. As flavinas também trabalham como coenzimas redox em muitas transformações biológicas e, conseqüentemente, vêm sendo empregadas como mediadores redox entre o eletrodo e várias enzimas ${ }^{11}$. Além disso, as flavinas são mediadores eficientes para a oxidação bioeletroquímica da nicotinamida adenina dinucleotídeo (NADH) ${ }^{12}$. Portanto, há um especial interesse no estudo da reação entre flavina com NADH ${ }^{13-15}$. Entretanto, o maior obstáculo encontrado neste tipo de estudo é a baixa diferença entre os valores de $E^{0^{\prime}}$ do NADH e da flavina livre, a qual resulta numa reação cineticamente lenta e termodinamicamente pouco favorável.

Através do desenvolvimento de novos eletrodos modificados, vários caminhos alternativos têm sido mostrados para mudar o $E^{0^{\prime}}$ de uma reação/catálise como resultado do processo de imobilização ${ }^{16-}$ ${ }^{17}$. Diferentes materiais de eletrodo estão sendo empregados, tais como diferentes materiais carbonáceos, ouro, platina, etc. ${ }^{18-20}$. O material do eletrodo usualmente afeta o $E^{0^{\prime}}$ das espécies eletroativas ${ }^{21}$, e a estabilidade depende do procedimento de imobilização ${ }^{22}$. Muitas tentativas estão sendo feitas para deslocar o $E^{0^{\prime}}$ dos compostos eletroativos para inserí-los dentro do intervalo de potencial considerado ideal $(-100$ a $0 \mathrm{mV})$, com o propósito de desenvolver biossensores ${ }^{21,23} \mathrm{e}$ aumentar a atividade eletrocatalítica.

*e-mail: kubota@iqm.unicamp.br
Os eletrodos de pasta de carbono são os mais utilizados, desde que estas superfícies são facilmente renovadas, apresentam baixa corrente de fundo e um amplo intervalo de potencial de trabalho ${ }^{24}$. Apesar disso, a incorporação de espécies em eletrodos de pasta de carbono ainda necessita ser melhorada, principalmente do ponto de vista dos aspectos de estabilidade e reprodutibilidade ${ }^{25}$.

Baseado neste contexto, materiais que permitam a imobilização de mediadores e moléculas biológicas são de grande potencial para o desenvolvimento de sensores e biossensores ${ }^{26}$. Vários trabalhos foram publicados ${ }^{27-29}$, utilizando zeólitas, argilas, fosfato de zircônio e sílica modificada como suportes para imobilização.

Atualmente, reagentes incorporados sobre a superfície de sílica gel e sílica sol-gel têm se tornado um procedimento comum para obtenção de suportes para catalisadore ${ }^{30,31}$, materiais para empacotar colunas em cromatografia líquida de alta eficiência ${ }^{32}$, adsorventes de íons metálicos ${ }^{33}$ e matrizes para imobilização de várias espécies, tais como enzimas ${ }^{34}$ e mediadores de elétron ${ }^{34}$. Esta performance faz da sílica uma excelente matriz a ser utilizada em eletrodos modificados. A sílica apresenta algumas características importantes, tais como rigidez, porosidade e estabilidade química, tornando-a matrizes promissoras para estes propósitos ${ }^{35}$.

$\mathrm{O}$ uso de sílica quimicamente modificada em uma interface eletrodo-solução tem atraído muita atenção, por explorar a versatilidade da sílica gel na imobilização de diferentes componentes, tais como espécies eletroativas. Sílica gel vem sendo modificada com óxidos metálicos, especialmente $\mathrm{Ti}(\mathrm{IV}), \mathrm{Zr}(\mathrm{IV}), \mathrm{V}(\mathrm{V})$, e $\mathrm{Nb}(\mathrm{V})$, resultando em materiais para imobilizações de mediadores de elétron ${ }^{30}$. As sílicas modificadas obtidas com óxidos metálicos ou fosfato são conhecidas como bons trocadores iônicos por causa da forte acidez da superfície do material.

Dentro deste contexto, este trabalho apresentará a preparação de eletrodos modificados com sílica gel modificada com óxido de nióbio (V) e riboflavina (RF) imobilizada, onde um planejamento fatorial foi aplicado visando otimizar este processo.

Deve-se mencionar que esta otimização é extremamente importante, uma vez que o processo de preparação dos eletrodos de pasta de carbono modificados com materiais inorgânicos apresenta alguns 
problemas, tais como a dificuldade de obtenção de uma pasta consistente e com propriedades condutoras. Desta forma, a quantidade de óleo mineral empregada e a proporção entre o grafite e a sílica modificada com o mediador são dois aspectos fundamentais para tal, além disso, a mistura entre os constituintes da pasta de carbono também é um importante aspecto a se considerar.

Os planejamentos quimiométricos ${ }^{36}$ são utilizados para maximizar ou minimizar algum tipo de resposta. Dentre os planejamentos mais utilizados estão os fatoriais $2^{\mathrm{k}}$, onde $\mathrm{k}$ corresponde ao número de fatores a serem estudados. Os planejamentos $2^{\mathrm{k}}$ permitem otimizar o sistema, com um número menor de experimentos quando comparados com os métodos univariados, além de tornarem possível a verificação dos efeitos de interação entre os fatores nos diferentes níveis desejados.

\section{PARTE EXPERIMENTAL}

\section{Reagentes}

Sílica gel (Fluka) com diâmetro médio dos poros de $6 \mathrm{~nm}$, área superficial específica de $520 \mathrm{~m}^{2} \mathrm{~g}^{-1}$, e tamanho de partícula de 0,05-0,2 nm, previamente ativada a $150{ }^{\circ} \mathrm{C}$ por $2 \mathrm{~h}$, foi usada. Grafite em pó (99,9\%, BDH), óleo mineral (Aldrich), pentacloreto de nióbio (Aldrich) e riboflavina (Sigma) foram utilizados sem tratamento prévio. Os demais reagentes empregados eram de grau analítico.

A preparação da sílica gel modificada com óxido de nióbio (SN), a adsorção da riboflavina (RF) sobre a SN (SNRF) e a caracterização deste material são apresentadas na literatura ${ }^{37,38}$.

\section{Otimização da preparação do eletrodo de trabalho por planejamento fatorial}

Um planejamento fatorial $2^{3}$ (estudo de 3 fatores em 2 níveis de resposta) foi empregado, a fim de otimizar a preparação do eletrodo de pasta de carbono contendo RF imobilizada.

Neste estudo, empregou-se a corrente de pico anódico $\left(\mathrm{Ip}_{\mathrm{a}}\right)$ como resposta para o planejamento fatorial $2^{3}$, a qual foi obtida através de voltametria cíclica (VC) no intervalo de potencial de -600 a $0 \mathrm{mV}$ a $v=20 \mathrm{mV} \mathrm{s}^{-1}$ em solução de $\mathrm{NaNO}_{3}(\mathrm{pH} 7,0)$.

Os fatores analíticos estudados foram proporção (quantidade de grafite e sílica modificada no eletrodo de pasta de carbono), mistura (ordem da mistura entre os constituintes da pasta de carbono) e concentração do eletrólito de suporte.

Para o fator proporção, os níveis estudados foram 50\% de grafite $/ 50 \%$ de SNRF (nível -) e 40\% de grafite/60\% de SNRF (nível +); para o fator mistura, empregaram-se dois procedimentos distintos, onde, no primeiro caso, misturou-se inicialmente grafite e SNRF, com posterior adição do óleo mineral (mistura 1, nível -) e no segundo, preparou-se inicialmente uma pasta com grafite e óleo mineral e, posteriormente, adicionou-se a SNRF (mistura 2, nível +). É importante mencionar que a quantidade de óleo mineral empregada na preparação dos eletrodos era fixa e no valor de $30 \mu \mathrm{L}^{38}$. Finalmente, para o fator concentração, as respostas foram obtidas em solução de $\mathrm{NaNO}_{3} 0,1 \mathrm{~mol} \mathrm{~L}^{-1}$ (nível -) e 0,5 mol L-1 (nível +).

A justificativa para a escolha destes fatores é que a proporção entre o grafite e a sílica modificada contendo o mediador imobiliza- do, como mencionado anteriormente, é importante na obtenção de uma pasta de carbono consistente e pouco resistiva.

Para o fator mistura, procurou-se verificar se a ordem de mistura dos reagentes influenciaria a resposta dos eletrodos. Para a mistura 1 procurou-se preencher as cavidades da sílica modificada (SNRF) com o grafite antes da adição do óleo mineral. Para a mistura 2, a prévia preparação de uma pasta entre o grafite e o óleo mineral com posterior adição da SNRF teve por objetivo a obtenção de uma pasta com uma distribuição mais homogênea da SNRF na superfície desta, o que poderia proporcionar maior intensidade de corrente.

O fator concentração de eletrólito foi escolhido, uma vez que este parâmetro pode afetar a reversibilidade da transferência de elétrons. Além disso, como a resposta escolhida neste estudo foi a intensidade de corrente anódica, esperava-se a obtenção de uma intensidade de corrente maior em soluções mais concentradas.

\section{Medidas eletroquímicas}

As medidas eletroquímicas foram realizadas com um potenciostato modelo PGSTAT 30 da Autolab, usando uma célula eletroquímica convencional de 3 eletrodos, com um eletrodo de calomelano saturado, ECS (Corning, St. Louis, USA) como eletrodo de referência, um fio de platina em forma de espiral como eletrodo auxiliar e o eletrodo de pasta de carbono modificado como eletrodo de trabalho.

\section{RESULTADOS}

\section{Características do material}

A Tabela 1 apresenta as características do material empregado, onde a quantidade de RF adsorvida sobre a superfície da sílica modificada, obtida por análise elementar, apresenta um resultado próximo daqueles observados na literatura ${ }^{39}$ para sistemas similares. Propõe-se que a interação ácido-base deva ser a responsável pela imobilização da RF sobre a superfície do material.

\section{Otimização da preparação do eletrodo de pasta de carbono por planejamento fatorial $2^{3}$}

O planejamento fatorial $2^{3}$ a fim de otimizar a preparação do eletrodo de pasta de carbono, foi realizado empregando a intensidade de corrente de pico anódico $\left(\mathrm{I}_{\mathrm{pa}}\right)$, uma vez que, no desenvolvimento de um sensor, o parâmetro analítico comumente empregado para a caracterização deste é a corrente de pico anódico (corrente de oxidação) em função da concentração do analito, como por exemplo, o NADH, e desta forma, justifica-se tal escolha.

A Tabela 2 apresenta os coeficientes de contrastes para um fatorial $2^{3}$, enquanto que a Tabela 3 mostra os resultados do planejamento fatorial $2^{3}$, onde o número entre parênteses é a ordem de realização dos ensaios. Para evitar a ocorrência de distorção estatística nos resultados, isto é, para impedir que erros atípicos sejam obrigatoriamente associados a determinadas combinações de níveis, os ensaios devem ser realizados em ordem totalmente aleatória ${ }^{36}$.

Conforme descrito na Parte Experimental, os fatores analíticos estudados foram a relação entre as quantidades de grafite e sílica

Tabela 1. Diâmetro médio dos poros, área superficial e quantidades de nióbio e Riboflavina (RF) adsorvidos sobre SN.

\begin{tabular}{lcccc}
\hline Material & Diâmetro médio dos poros $(\mathrm{nm})$ & Área superficial $\left(\mathrm{m}^{2} \mathrm{~g}^{-1}\right)$ & $\mu$ mol de $\mathrm{Nb} / \mathrm{g}$ de material & mmol de RF $/ \mathrm{g}$ de material $^{*}$ \\
\hline $\mathrm{SN}$ & 6,0 & $496 \pm 7^{*}$ & $1400 \pm 20$ & $27 \pm 1$ \\
\hline
\end{tabular}

* Os resultados apresentados nesta Tabela foram obtidos em triplicata e são estimativas do desvio padrão 
Tabela 2. Coeficientes de contraste para um planejamento fatorial $2^{3}$

\begin{tabular}{ccccc}
\hline & & & $(-)$ & $(+)$ \\
Fatores: & 1: Proporção (\%) & & $50 / 50$ & $40 / 60$ \\
& 2: Mistura (tipo) & & 1 & 2 \\
& 3: Concentração $\left(\mathrm{mol} \mathrm{L}^{-1}\right)$ & 0,1 & 0,5 \\
\hline Ensaio & Fator 1 & Fator 2 & Fator 3 \\
\hline 1 & - & - & - \\
2 & + & - & - \\
3 & - & + & - \\
4 & + & + & - \\
5 & - & - & + \\
6 & + & - & + \\
7 & - & + & + \\
8 & + & + & \\
\hline
\end{tabular}

Tabela 3. Resultados do planejamento fatorial $2^{3}$

\begin{tabular}{ccccc}
\hline Ensaio & $\begin{array}{c}\text { Resposta } 1 * \\
\left(\mathrm{I}_{\mathrm{pa}} / \mu \mathrm{A}\right)\end{array}$ & $\begin{array}{c}\text { Resposta } 2 * \\
\left(\mathrm{I}_{\mathrm{pa}} / \mu \mathrm{A}\right)\end{array}$ & $\begin{array}{c}\text { Média } \\
\left(\mathrm{I}_{\mathrm{pa}} / \mu \mathrm{A}\right)\end{array}$ & $\begin{array}{c}\text { Estimativa do } \\
\text { desvio padrão } \\
\left(\mathrm{I}_{\mathrm{pa}} / \mu \mathrm{A}\right)\end{array}$ \\
\hline 1 & $0(\mathbf{4})$ & $0(\mathbf{9})$ & 0 & 0 \\
2 & $1,72(\mathbf{5})$ & $2,10(\mathbf{1 3 )}$ & 1,91 & 0,27 \\
3 & $0,64(\mathbf{1 2})$ & $0,66(\mathbf{1})$ & 0,65 & 0,01 \\
4 & $1,15(\mathbf{3})$ & $1,17(\mathbf{1 6})$ & 1,16 & 0,01 \\
5 & $0(\mathbf{1 0})$ & $0(\mathbf{2})$ & 0 & 0 \\
6 & $2,04(\mathbf{6})$ & $2,41(\mathbf{1 1})$ & 2,23 & 0,26 \\
7 & $0,76(\mathbf{7})$ & $0,66(\mathbf{1 5})$ & 0,71 & 0,07 \\
8 & $1,63(\mathbf{8})$ & $1,44(\mathbf{1 4})$ & 1,54 & 0,13 \\
\hline
\end{tabular}

* Os experimentos foram realizados em duplicata

modificada contendo a RF imobilizada (denominado fator proporção), a forma de preparação dos eletrodos (fator mistura) e a concentração do eletrólito suporte (fator concentração).

A Figura 1 ilustra que a melhor condição para a obtenção do sinal $\left(\mathrm{I}_{\mathrm{pa}}\right)$ está nos níveis $(+-+)$, o que corresponde à proporção $40 \%$ de grafite: $60 \%$ de SNRF, tipo de mistura 1 (grafite + SNRF/óleo mineral) e concentração de eletrólito $0,5 \mathrm{~mol} \mathrm{~L}^{-1}$. Isto demonstra que a resposta aumenta quando a RF está em maior proporção na pasta, o óleo mineral deve ser adicionado por último na preparação do eletrodo de pasta de carbono e em solução de eletrólito mais concentrada.

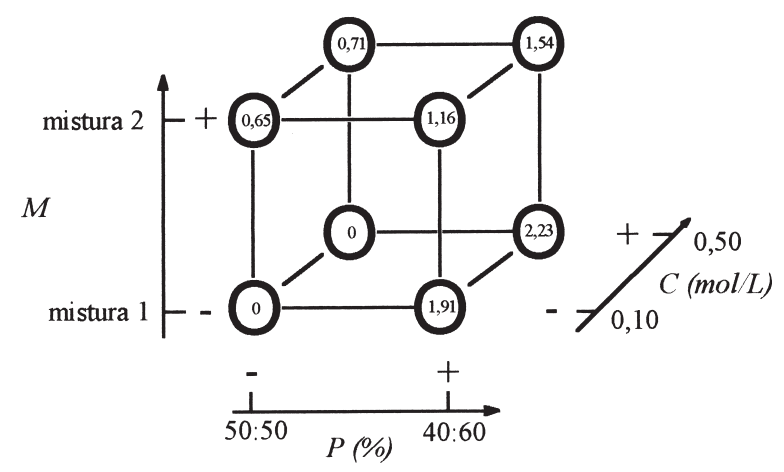

Figura 1. Diagrama para interpretação dos efeitos proporção, mistura e concentração do eletrólito no planejamento $2^{3}$. Os valores apresentados neste diagrama representam a média das resposta de $I_{p a}$ para cada ensaio

\section{Caracterização eletroquímica}

Os voltamogramas cíclicos obtidos durante esta etapa de otimização foram caracterizados eletroquimicamente através do estudo de $\Delta \mathrm{E}_{\mathrm{p}}$ e da relação $\mathrm{I}_{\mathrm{pa}} / \mathrm{I}_{\mathrm{pc}}$, de onde se confirmou a discussão feita anteriormente, ou seja, o eletrodo preparado nas condições ótimas, apresentou $\Delta \mathrm{E}_{\mathrm{p}}=55 \mathrm{mV}$ e $\mathrm{I}_{\mathrm{pa}} / \mathrm{I}_{\mathrm{pc}}=0,98$, enquanto que para um outro eletrodo, preparado em condição experimental diferente, estes valores foram $\Delta \mathrm{E}_{\mathrm{p}}=60 \mathrm{mV}$ e $\mathrm{I}_{\mathrm{pa}} / \mathrm{I}_{\mathrm{pc}}=6,33$ (reversibilidade muito baixa). Estes voltamogramas estão ilustrados na Figura 2.

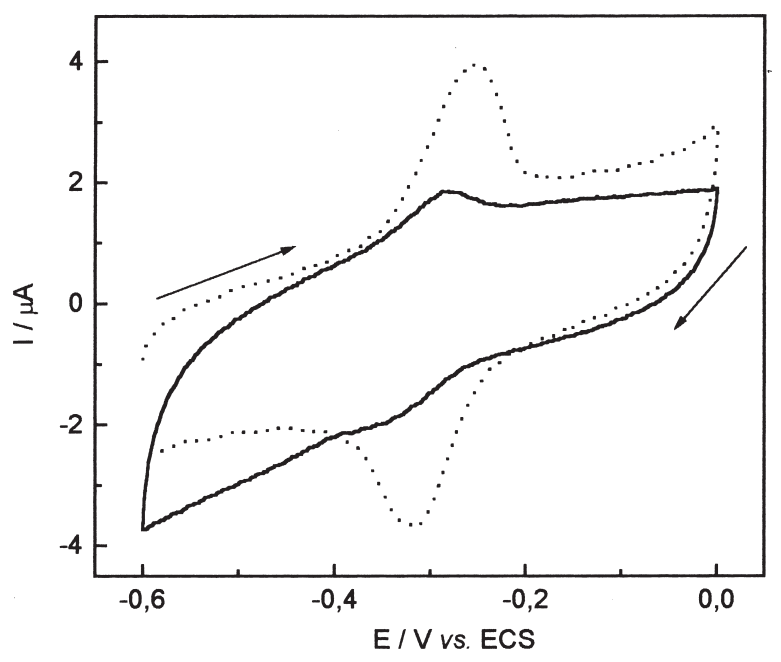

Figura 2. Voltamogramas cíclicos correspondentes aos ensaios 6, onde as condições foram proporção $60 \%$ de SNRF e $40 \%$ de grafite, mistura tipo 1 e concentração do eletrólito $0,5 \mathrm{~mol} \mathrm{L^{-1 }}$ (‥) e 7, onde as condições foram proporção $50 \%$ de SNRF e $50 \%$ de grafite, mistura tipo 2 e concentração do eletrólito $0,5 \mathrm{~mol} \mathrm{~L}^{-1}(-)$ para o planejamento fatorial $2^{3}$

Estes dados são importantes e demonstram que, embora o parâmetro analítico utilizado como resposta durante esta etapa de otimização da preparação do eletrodo de trabalho tenha sido a $I_{\mathrm{pa}}$, foi possível, otimizar também o parâmetro eletroquímico $\Delta \mathrm{E}_{\mathrm{p}}$, o qual é evidente na discussão realizada sobre a Figura 2.

A partir desta verificação, os eletrodos foram preparados nas condições experimentais do ensaio 6, a fim de se estudar as propriedades eletroquímicas da RF imobilizada sobre diferentes matrizes ${ }^{38}$.

\section{Tratamento estatístico das respostas}

\section{Cálculo dos efeitos}

A Tabela 2 contém todos os sinais necessários para o cálculo dos efeitos principais e de interações (12; 13; 23 e 123). O divisor é 8 para a média, uma vez que se emprega apenas sinais positivos no cálculo deste parâmetro, e 4 para cada um dos efeitos. Empregando os sinais apropriados como coeficientes das respostas médias observadas e em seguida aplicando os divisores, calculou-se os sete efeitos e a média global. Em todos os cálculos foram utilizadas todas as respostas.

Qualquer efeito, portanto, pode ser interpretado como a diferença entre duas médias, cada uma das quais contendo metade das observações. Através de uma matriz 8x8 (denominada matriz X) e com os sinais algébricos dos coeficientes de contraste com elementos +1 e -1, os efeitos serão dados, a menos dos divisores, pelo produto $\mathrm{X}^{\mathrm{t}} \mathrm{y}$, onde $\mathrm{X}^{\mathrm{t}}$ é a matriz transposta de $\mathrm{X}$ e $\mathrm{y}$, o vetor coluna contendo as respostas médias dos ensaios. 
Foram calculados os erros padrão dos efeitos através das equações apresentadas na literatura ${ }^{36}$, e o valor obtido foi de aproximadamente 0,072 . O erro padrão da média será a metade disso, 0,036, porque os coeficientes da combinação linear nesse caso são todos iguais a $1 / 8$, ao invés de $\pm 1 / 4^{36}$. A Tabela 4 mostra os valores calculados para todos os efeitos e seus erros padrão.

Tabela 4. Efeitos calculados para o planejamento fatorial $2^{3}$ e seus erros padrão

Média: $1,024 \pm 0,036$

Efeitos Principais:

1 (Proporção)

$1,367 \pm 0,072$

2 (Mistura)

$-0,022 \pm 0,072$

3 (Concentração)

$0,187 \pm 0,072$

Interação de 2 fatores

12

$-0,702 \pm 0,072$

13

$0,157 \pm 0,072$

23

$0,027 \pm 0,072$

Interação de 3 fatores

123

$-0,002 \pm 0,072$

A resposta deste sistema $\left(\mathrm{I}_{\mathrm{pa}}\right)$ pode ser representada através de um modelo que, em quimiometria, é chamado de modelo estatísti$\mathrm{co}^{36}$. Através deste modelo calcularam-se as estimativas dos coeficientes (b) através da equação :

$\mathrm{b}=(1 / 8) \mathrm{X}^{\mathrm{t}} \mathrm{y}$

Para saber-se quais os valores, obtidos através desta equação, são significativamente diferentes de zero, empregou-se a distribuição de Student ${ }^{36}$ para testar a significância dos valores estimados. O intervalo de confiança é dado por:

$\mathrm{b} \pm \mathrm{t}_{\mathrm{n}}($ erro padrão de $\mathrm{b})$

Neste caso, onde temos 8 ensaios (planejamento fatorial $2^{3}$ ) e com um intervalo de confiança de $95 \%$, o valor de $\mathrm{t}_{8,(95 \%)}$ (Student test) é igual a 2,306, sendo este um valor tabelado ${ }^{36}$.

O erro padrão da estimativa é dado por:

$\mathrm{V}(\mathrm{b})=\left(\mathrm{X}^{\mathrm{t}} \mathrm{X}\right)^{-1} \mathrm{~s}^{2}$

Através das equações anteriores foram efetuados os cálculos e a equação que melhor descreve este modelo é a seguinte:

$Y=1,02( \pm 0,05)+0,68( \pm 0,05) X_{1}-0,35( \pm 0,05) X_{1} X_{2}$

onde $\mathrm{Y}$ é a resposta, $\mathrm{X}_{1}$ e $\mathrm{X}_{2}$ são as variáveis codificadas correspondentes à proporção e mistura, enquanto que os valores numéricos indicam, respectivamente, a média das respostas, a estimativa do efeito proporção e a estimativa da interação proporção/mistura. Os valores entre parênteses são os erros padrão das estimativas.

Portanto, através do tratamento estatístico dos dados obtidos durante a otimização da preparação do EPC modificado, concluiu-se que apenas o fator 1 (proporção) e a interação 12 (proporção/mistura) são estatiscamente significativos, com intervalos de confiança de $95 \%$.

Finalmente, através deste planejamento fatorial foi possível observar que um efeito de interação de 2 fatores é estatisticamente significativo, o qual não é possível de ser observado em um estudo univariado. Desta forma, este procedimento deveria ser recomendado para estudos e otimização de eletrodos quimicamente modificados.
Além disso, embora a Figura 1 mostre que a resposta do eletrodo $\left(\mathrm{I}_{\mathrm{pa}}\right)$ seja melhor para soluções de eletrólito mais concentradas, este fator não é estatisticamente significativo sendo permitido, desta forma, se trabalhar em concentrações menos concentradas.

\section{CONCLUSÕES}

Através do planejamento fatorial realizado com o objetivo de otimizar a preparação dos eletrodos de pasta de carbono contendo SNRF, foi possível concluir que o fator proporção e a interação proporção/mistura são estatisticamente significativos. O efeito de interação observado aqui poderia ser indicativo de que efeitos de interação são importantes para sistemas deste tipo.

A caracterização eletroquímica destes eletrodos $\left(\mathrm{I}_{\mathrm{pa}} / \mathrm{I}_{\mathrm{pc}} \mathrm{e} \Delta \mathrm{E}_{\mathrm{p}}\right)$, juntamente com a interpretação estatística dos resultados, mostrou que o melhor procedimento de preparação destes é com proporção $60 \%$ de SNRF e $40 \%$ de grafite e a mistura prévia dos constituintes sólidos (SNRF e grafite em pó), com posterior adição de óleo mineral.

Em geral, a repetibilidade obtida para estes sistemas otimizados é excelente, apresentando valores de desvio padrão médio relativo (rds) em torno de $2 \%$ para $\mathrm{n}=10$.

\section{AGRADECIMENTOS}

Os autores agradecem à FAPESP pelo suporte financeiro.

\section{REFERÊNCIAS}

1. Katz, E.; Lotzbeyer, T.; Schlereth, D. D.; Schuhmann, W.; Schmidt, H-L.; J. Electroanal. Chem. 1994, 373, 189.

2. Ni, F.; Feng, H.; Gorton, L.; Cotton, T. M.; Langmuir 1990, 6, 66.

3. Nagy, G.; Kapui, I.; Gorton, L.; Anal. Chim. Acta 1995, 305, 65.

4. Domínguez, E.; Lan, H. L.; Okamoto, Y.; Hale, P. D.; Skotheim, T. A.; Gorton, L.; Hahnhagerdal, B.; Biosens. Bioelectron. 1993, 8, 229.

5. Fernandes, J. C. B.; Oliveira Neto, G.; Rohwedder, J. J. R.; Kubota, L. T.; J. Braz. Chem. Soc. 2000, 11, 349.

6. Gorton, L.; Domínguez, E.; Electroanalysis, Rev. Mol. Biotechnol. 2002, 82,371 .

7. Sun, W. L.; Kong, J. L.; Deng, J. Q.; Anal. Lett. 1996, 29, 2425.

8. De Lucca, A. R.; Santos, A. S.; Pereira, A. C.; Kubota, L. T.; J. Colloid Interface Sci. 2002, 254, 113.

9. Friedman, R. M.; J. Electroanal. Chem. 1999, 472, 147.

10. Cosnier, S.; Décout, J-L.; Fontecave, M.; Frier, C.; Innocent, C.; Electroanalysis 1998, 10, 521

11. Ogino, Y.; Takagi, K.; Kano, K.; Iketa, T.; J. Electroanal. Chem. 1995, 396, 517

12. Chi, Q-J.; Dong, S-J.; J. Mol. Catal. A: Chem. 1996, 105, 193.

13. Birss, V. I.; Guha-Thakurta, S.; McGarvey, C. E.; Quach, S.; Vanýsek, P.; J. Electroanal. Chem. 1997, 423, 13.

14. Cosnier, S.; Fontecave, M.; Limosin, D.; Nivière, V.; Anal. Chem. 1997, $69,3095$.

15. Gergel, A.; Comtat, M.; J. Electroanal. Chem. 1991, 302, 219.

16. Gorton, L.; Electroanalysis 1995, 7, 23.

17. Santos, A. S.; Gorton, L.; Kubota, L. T.; Electrochim. Acta 2002, 47, 3351.

18. Yamashita, M.; Rosatto, S. S.; Kubota, L. T.; J. Braz. Chem. Soc. 2002, 13,635 .

19. Coche-Guerente, L.; Cosnier, S.; Inocent, C.; Mailley, P.; Anal. Chim. Acta 1995, 311, 23 .

20. Coche-Guerente, L.; Deronzier, A.; Mailley, P.; Moutet, J. C.; Anal. Chim. Acta 1994, 289, 143.

21. Pogorelova, S. P.; Zayats, M.; Kharitonov, A. B.; Katz, E.; Willner, I.; Sens. Actuators, B 2003, 89, 40 .

22. Munteanu, F. D.; Kubota, L. T.; Gorton, L.; J. Electroanal. Chem. 2001, $509,2$.

23. Gorton, L.; Persson, B.; Hale, P. D.; Boguslavsky, L. I.; Karan, H. I.; Lee, H. S.; Skotheim, T. A.; Lan, H. I.; Okamoto, Y. Em Biosensors and Chemical Sensors - Optimizing Performance Through Materials; Edelman, P. G.; Wang, J. O., eds.; ACS Symposium Series, American Chemical Society: Washington, D. C., 1992, vol. 487, p. 56.

24. Kalcher, K.; Kauffmann, J. M.; Wang, J.; Svancara, I.; Vytras, K.; Neuhold, C.; Yang, Z.; Electroanalysis 1995, 7, 5. 
25. Mullor, S. G.; Sánchez-Cabezudo, M.; Ordieres, A. J. M.; Ruiz, B. L.; Talanta 1996, 43, 779.

26. Gorton, L.; Karan, H. I.; Hale, P. D.; Inagaki, T.; Okamoto, Y.; Skotheim, T. A.; Anal. Chim. Acta 1990, 228, 23.

27. Palecek, E.; Electroanalysis 1996, 8, 7.

28. Kubota, L. T.; Gushikem, Y.; Perez, J.; Tanaka, A. A.; Langmuir 1995, 11, 1009.

29. Rolison, D. R.; Chem. Rev. 1990, 90, 867.

30. Gushikem, Y.; Rosatto, S. S.; J. Braz. Chem. Soc. 2001, 6, 695.

31. Alfaya, A. A. S.; Kubota, L. T.; Quim. Nova 2002, 25, 835.

32. Durrani, A. A.; Garret, A.; Johnson, R. A.; Sood, S. K.; Tyman, J. H. P.; J. Liq. Chromatogr. Relat. Technol. 2002, 25, 1543.
33. Mahmoud, M. E.; Al Saadi, M. S. M.; Anal. Chim. Acta 2001, 450, 239.

34. Walcarius, A.; Electroanalysis 1998, 10, 18.

35. Katakis, I.; Domínguez, E.; Mikrochim. Acta 1997, 126, 11.

36. Neto, B. B.; Scarmínio, I. S.; Bruns, R. E.; Planejamento e Otimização de Experimentos, $2^{\mathrm{a}}$ ed., Campinas, 1996.

37. Denofre, S.; Gushikem, Y.; Davanzo, C. U.; J. Solid State Inorg. Chem. 1991, 28, 1295.

38. Pereira, A. C.; Santos, A. S.; Kubota, L. T.; J. Colloid Interface Sci. 2003, $265,351$.

39. Santos, A. S.; Pereira, A. C.; Kubota, L. T.; J. Braz. Chem. Soc. 2002, 13, 495. 